\title{
A Note on Multiple Flow Equilibria
}

\author{
S. J. JACOBS ${ }^{1}$
}

\begin{abstract}
A set of ordinary differential equations describing a mechanical system subject to forcing and dissipation is considered. A topological argument is employed to show that if all time-dependent solutions of the governing equations are bounded, the equations admit $N$ steady solutions, where $N$ is a positive odd integer and where at least $(N-1) / 2$ of the steady solutions are unstable. The results are discussed in the context of atmospheric flows, and it is shown that truncated forms of the quasigeostrophic equations of dynamic meteorology and of Budyko-Sellers climate models satisfy the hypotheses of the theorem.
\end{abstract}

Key words: Dynamic systems, multiple equilibrium, stability.

\section{Introduction}

Several years ago Charney and DeVore (1979), Vickroy and Dutton (1979), and WIIN-NiELSEN (1979) found that the equations governing a low order barotropic system with forcing and dissipation admit multiple steady solutions. For this system the governing equations admit $N$ steady solutions, where $N$ is a positive odd integer and where at least $(N-1) / 2$ of the steady solutions are unstable. The same pattern is found in treatments of Budyko-Sellers climate models (e.g., NORTH et al., 1979), in the study of open channel flow over a corrugated bottom (ZHU, 1988), and in other problems of interest in atmospheric science and fluid mechanics.

Although in many cases steady solutions of the governing equations can be found analytically, in general the search for equilibrium solutions must be carried out numerically. Path following methods have been developed to treat the dependence of equilibrium points on a parameter (KELLER, 1979), and techniques have been proposed to find all the solutions of a system of polynomial equations (WRIGHT, 1985). Carrying out these procedures is often tedious and time-consuming, and therefore qualitative information about the number of solutions can be extremely useful.

The purpose of this note is to show that the results discussed above for steady solutions hold for all truncated models for which transient solutions of the

\footnotetext{
'Department of Atmospheric, Oceanic, and Space Sciences, Department of Mechanical Engineering and Applied Mechanics, The University of Michigan, Ann Arbor, MI 48109, USA.
} 
governing equations are bounded. We also provide information concerning the stability of equilibrium solutions when the solution curve exhibits limit points. Our main result is given by a theorem in Section 2. This is followed by a proof that truncated forms of the quasi-geostrophic equations of dynamic meteorology and of Budyko-Sellers climate models satisfy the hypotheses of the theorem, and by a discussion of the results.

\section{Analysis}

To avoid the advanced topological analysis needed for treating partial differential equations (e.g., BENJAMIN, 1976), we reduce the governing equations of a dynamical system to a set of ordinary differential equations by use of a truncated spectral expansion. Hence, letting the components of $\mathbf{x}(t)$ denote the time-dependent amplitudes in a spectral expansion, we consider the system of ordinary differential equations

$$
\frac{d \mathbf{x}}{d t}+\mathbf{f}(\mathbf{x}, \lambda)=0,
$$

where the constant vector $\lambda$ represents the parameters and $\mathbf{x}$ and $\mathbf{f}$ are $n$-dimensional vectors. For future reference we define the Jacobian matrix $J_{i k}$ and the corresponding Jacobian determinant $J$ by $J_{i k}=\partial f_{i} / \partial x_{k}$ and $J=\operatorname{det}\left(J_{i k}\right)$.

In the present study we assume that $f$ is a differentiable function of its arguments with a convergent power series expansion in $\left(\mathbf{x}-\mathbf{x}_{m}\right)$ near all points $\mathbf{x}_{m}$ for which $f$ vanishes, that the equations

$$
\mathbf{f}=0, \quad J=0,
$$

are satisfied only when $\lambda$ lies on a discrete number of bifurcation surfaces, that a finite positive number $r$ exists for which

$$
\mathbf{x} \cdot \mathbf{f}>0
$$

on $|\mathbf{x}| \geqslant r$, and that $\mathbf{f} \neq 0$ for $|\mathbf{x}|=r$. Since

$$
\frac{d}{d t}(\mathbf{x} \cdot \mathbf{x})=-2(\mathbf{x} \cdot \mathbf{f}),
$$

it follows that all solutions of (1) are bounded.

The main result of the present paper is as follows:

Theorem. Let the above hypotheses be satisfied. Then

1) Eq. (1) has at least one equilibrium solution;

2) if $J \neq 0$ at the equilibrium solutions, the number $N$ of such solutions is odd, i.e. $N=(2 M+1)$, where $M$ is zero or a positive integer;

3) if $J \neq 0$ at the equilibrium solutions, at least $M$ of the equilibrium solutions are unstable; 
4) if $N=3$ and if a graph of the equilibrium solution for $|\mathbf{x}|$ as a function of one of the components of $\lambda$ has an $S$ shape, the solution on the middle branch of the $S$ shaped curve is unstable.

The proof of the theorem is topological and requires some facts about the Brouwer degree of a mapping (BERGER, 1977; Lloyd 1978). Let $\mathbf{f}(\mathbf{x})$ be a continuous function, $\Omega$ a bounded open set in $R^{n}$, and $\partial \Omega$ the boundary of $\Omega$. Then, letting $\epsilon$ denote set membership, there exists an integer valued function $d(f, \Omega)$, the Brouwer degree, which is defined if $\mathbf{f} \neq 0$ for $\mathbf{x} \in \partial \Omega$, and which has the following properties:

1) $d(\mathbf{f}, \Omega) \neq 0$ implies that $\mathbf{f}=0$ has at least one solution $\mathbf{x} \in \Omega$;

2) if the solutions $\mathbf{x}_{m}$ of $\mathbf{f}=0$ are isolated, the index $i_{m}$ is defined as $d\left(\mathbf{f}, \Omega_{m}\right)$, where $\Omega_{m}$ is a neighborhood of $\mathbf{x}_{m}$ containing no other solutions, and

$$
d(\mathbf{f}, \Omega)=\Sigma i_{m} ;
$$

3) if $\mathbf{f}$ is differentiable at $\mathbf{x}_{m}$ and $J\left(x_{m}\right) \neq 0$, the index $i_{m}=(-1)^{v}$, where $v$ is the number, counting multiplicity, of the real negative eigenvalues of $J_{i k}\left(\mathbf{x}_{m}\right)$;

4) if $s$ is a scalar parameter lying in a closed interval $S$ of the real axis and $\mathbf{h}(\mathbf{x}, s)$ is a continuous function of its arguments, then $d(\mathbf{h}(\mathbf{x}, s), \Omega)$ is independent of $s$ if $\mathbf{h} \neq 0$ for $s \in S$ and $\mathbf{x} \in \partial \Omega$.

To prove the theoren, we let $\Omega$ denote the open sphere $|\mathbf{x}|<r$ and note that the function

$$
\mathbf{h}(\mathbf{x}, s)=s \mathbf{x}+(1-s) \mathbf{f}, \quad s \in[0,1],
$$

satisfies

$$
\mathbf{x} \cdot \mathbf{h}=s r^{2}+(1-s)(\mathbf{x} \cdot \mathbf{f})<0
$$

for $\mathbf{x} \in \partial \Omega, s \in[0,1]$ and so $\mathbf{h} \neq 0$ for $\mathbf{x} \in \partial \Omega, s \in[0,1]$. According to Properties 2) and 3) of the Brouwer degree $d(\mathbf{x}, \Omega)=1$, and hence, by Property 4),

$$
d(\mathbf{f}, \Omega)=d(\mathbf{h}(\mathbf{x}, 0), \Omega)=d(\mathbf{h}(\mathbf{x}, 1), \Omega)=d(\mathbf{x}, \Omega),
$$

which in turn yields

$$
d(\mathbf{f}, \Omega)=1 .
$$

The first conclusion of the theorem follows from (8) and Property 1) of the Brouwer degree. The second follows from (8) and Properties 2) and 3); the sum of the indices is unity, each index takes on the values 1 or -1 , and this implies that the number $N$ of solutions of $\mathbf{f}=0$ is odd, $N=(2 M+1)$, where $M$ of the solutions have index -1 and $(M+1)$ have index 1 . The third conclusion follows from the proof of the second and Property 3), since an index $i_{m}=-1$ implies that $J_{i k}\left(\mathbf{x}_{m}\right)$ has at least one real negative eigenvalue, which in turn implies, by the principle of stability in the first approximation (HAHN, 1967, Th. 28.1, p. 122) that $\mathbf{x}_{m}$ is unstable. 


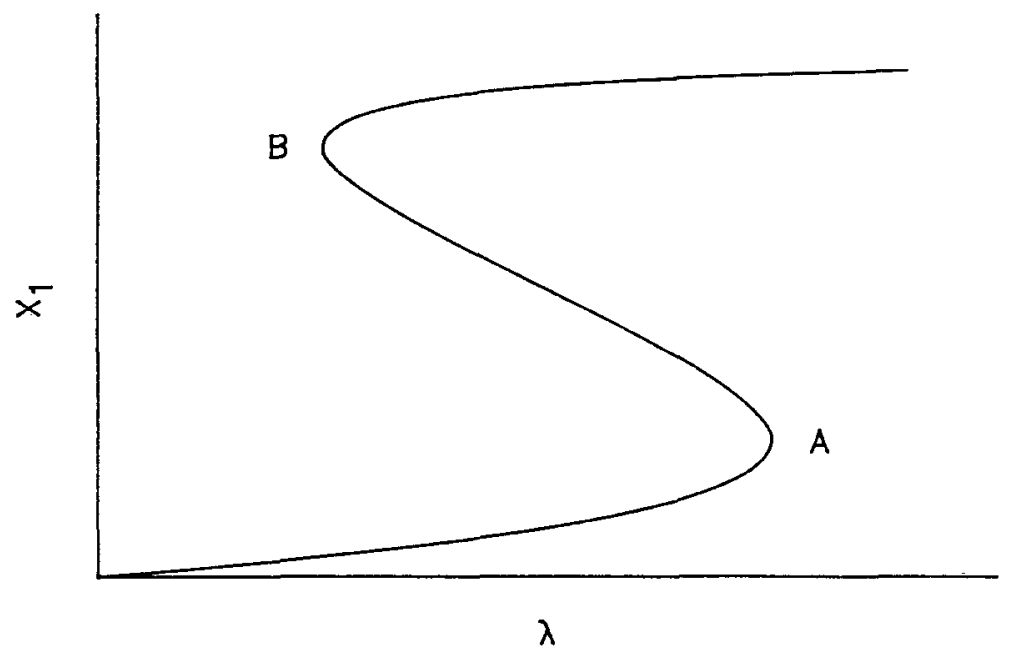

Figure 1

Schematic diagram of a component $x_{1}$ of the state vector as a function of a parameter $\lambda$. The branch $A B$ is unstable.

The fourth conclusion of the theorem follows from the fact that if solutions of $\mathbf{f}=\mathbf{0}$ appear as $\lambda$ passes through a critical value, they must occur in pairs and have indices of opposite signs. Consequently, if a graph of the equilibrium solution for $|\mathbf{x}|$ as a function of one of the parameters has an $S$ shape, as shown in Figure 1, the indices of solutions on two different branches of the curve have opposite signs. Since the sum of the indices of solutions on the lower, middle, and upper branches of the $S$ shaped curve must be unity, by (8), the index of a solution on the middle branch is -1 , and so this solution is unstable.

\section{Applications}

In a study of quasi-geostrophic flow on the sphere, DutTon (1976) showed that with certain simplifying assumptions concerning dissipation, the boundary condition at the ground, and the boundary condition at the top of the atmosphere, the governing equations for a spectral model describing either baroclinic or barotropic flow can be expressed in the form

$$
\frac{d x_{i}}{d t}+A_{i j k} x_{j} x_{k}+B_{i j} x_{j}-C_{i}=0,
$$

where the summation convention is applied and all indices run from 1 to $n$, in which $n$ is finite but arbitrary. Here the components of $\mathbf{x}$ are the expansion coefficients in an eigenfunction expansion for which the energy of the system is proportional to 


$$
E=\mathbf{x} \cdot \mathbf{x},
$$

and $A_{i j k}, B_{i j}$ and $C_{i}$ are constants.

It is easily shown from Dutton's equations that

$$
A_{i j k} x_{i} x_{j} x_{k}=0,
$$

for all $\mathbf{x}$ if the energy of the system is represented by (10), and that the matrix

$$
R_{i j}=B_{i j}+B_{j i}
$$

is positive definite. The equations studied by Charney and deVore, Vickroy and Dutton, and Wiin-Nielsen are also of the form (9), and, by a change of variable, can be shown to satisfy (11) and (12).

The set of equations (9) is a forced dissipative system according to Lorenz's definition (LoRENZ, 1963), and therefore, defining the vector $\mathbf{e}$ as the solution of

$$
R_{i j} e_{j}=C_{i},
$$

it follows from a calculation given by Lorenz that

$$
\frac{d E}{d t}=R_{i j} e_{i} e_{j}-R_{i j}\left(x_{i}-e_{i}\right)\left(x_{j}-e_{j}\right),
$$

which in turn shows that all solutions of (9) eventually lie on or within an ellipsoid in phase space. Accordingly, the hypotheses of Section 2 are verified for this model, and all equilibrium solutions of Dutton's truncated equations and of truncated barotropic systems satisfy our main theorem. Consequently, the solution pattern found in the papers by Charney and deVore, Vickroy and Dutton, and WiinNielsen is independent of the order of the truncation. This conclusion holds also for quasi-geostrophic two-layer models with linear viscous forces and for the convection equations treated in Dutton's text (DuTTON, 1986, Sections 15.2.2-15.2.5).

As a second example, we consider Budyko-Sellers climate models using the variational formulation given in the paper by NORTH et al. cited earlier. Let $t$ denote time, $\mu$ the sine of the latitude, $C$ a heat capacity, $T(\mu, t)$ the temperature, and $F[T]$ a functional of the temperature defined by NORTH et al. Then, as shown by NORTH et al., if $T$ is expanded in the form

$$
T=x_{0}(t) P_{0}(\mu)+x_{2}(t) P_{2}(\mu)+\cdots,
$$

where $P_{n}(\mu)$ is a Legendre polynomial, the amplitudes $x_{n}(t)$ satisfy

$$
\frac{d x_{n}}{d t}+\frac{\partial F}{\partial x_{n}}=0, \quad n=0,1, \ldots
$$

Here $F$ can be approximated by

$$
F\left[x_{0} P_{0}+x_{2} P_{2}+\cdots\right]=\sum_{n_{\text {even }}} a_{n}\left(x_{n}\right)^{2}
$$


for large $x_{n}$, where the constants $a_{n}$ are positive, and substituting (17) into (16) shows that the hypotheses introduced in Section 2 are also satisfied for this model. Hence, as before, the solution pattern is given by the theorem of Section 2. This result also holds in the analytically intractable model for which the step function behavior of the albedo on temperature usually assumed in climate models is replaced by a more realistic smooth dependence (GRIFFEL and DRAZIN, 1981), and is consistent with Griffel and Drazin's numerical calculations.

\section{Discussion}

The aim of the work reported here is to prove that the results on multiple flow equilibria obtained in previous studies of forced atmospheric flows and of BudykoSellers climate models are generic, and that the solution pattern for the equilibrium points discovered in these studies holds for any system for which the transient solutions are intrinsically bounded. This aim is accomplished by the theorem of Section 2 and by the proof in Section 3 that the equations describing quasigeostrophic flow and Budyko-Sellers climate models satisfy the hypotheses of the theorem. The information provided by the theorem can be used in a variety of ways as a guide in carrying out numerical calculations of the equilibrium points. For example, a numerical treatment of a system satisfying the hypotheses of the theorem which yields an even number of equilibrium solutions is necessarily incorrect or incomplete.

As regards mathematical originality, the last conclusion of the theorem of Section 2 was proved by SATTINGER (1972) on the hypothesis that the lower and upper branches of the $S$ shaped curve are stable for some values of the parameters. The present theorem is proved under the less restrictive hypothesis that the indices of these solutions are +1 . The first three conclusions of the theorem are the same as those of Theorem 1.7.2 in a book by GaVALAS (1968) on chemically reacting systems. In his proof Gavalas imposes restrictions on the components of his state vector which insure that all chemical concentrations remain nonnegative. We have replaced this assumption by the hypothesis that the solutions of (1) remain bounded, which is more natural for a mechanical system and which is verified in the applications treated in Section 3.

\section{REFERENCES}

BENJAMIN, T. B. (1976), Applications of Leray-Schauder degree theory to problems of hydrodynamic stability, Proc. Camb. Phil. Soc. 79, 373-392.

Berger, M. S., Nonlinearity and Functional Analysis (Academic Press, New York 1977).

Charney, J. G. and DeVore, J. G. (1979), Multiple flow equilibria in the atmosphere and blocking, J. Atm. Sci. 36, 1205-1216. 
Dutron, J. A. (1976), The nonlinear quasi-geostrophic equation. Part II: Predictability, recurrence and limit properties of thermally-forced and unforced flows, J. Atm. Sci. 33, 1431-1453.

Dutton, J. A., The Ceaseless Wind: An Introduction to the Theory of Atmospheric Motion (Dover Publications, New York 1986).

Gavalas, G. R., Nonlinear Differential Equations of Chemically Reacting Systems (Springer-Verlag, New York 1968).

Griffel, D. H. and Drazin, P. G. (1981), On diffusive climatological models, J. Atm. Sci. 38, 2327-2332.

HaHN, W., Stability of Motion (Springer-Verlag, New York 1967).

KeLler, H. B., Global homotopies and Newton methods, In Recent Advances in Numerical Analysis (eds. C. de Boor and G. H. Golub) (Academic Press, New York 1979) pp. 73-94.

North, G. N., Howard, L., Pollard, D., and Wielicki, B. (1979), Variational formulation of Budyko-Sellers climate models, J. Atm. Sci. 36, 255-259.

Lloyd, N. G., Degree Theory (Cambridge University Press, London and New York 1978).

LoRenz, E. N. (1963), Deterministic nonperiodic flow, J. Atm. Sci. 20, 130-141.

SATTINGER, D. H. (1972), Stability of solutions of nonlinear equations, Math. Anal. and Applic. 39, 1-12.

Vickroy, J. G. and DutTon, J. A. (1979), Bifurcation and catastrophe in a simple forced quasigeostrophic flow, J. Atm. Sci. 36, 42-52.

WIIN-NIELSEN, A. C. (1979), Steady states and stability properties of a low-order barotropic system with forcing and dissipation, Tellus 31, 375-386.

WRIGHT, A. H. (1985), Finding all solutions to a system of polynomial equations, Math. Comp. 44, $125-133$

ZHU, S., Open Channel Flow Near the Resonance Speed (University of Michigan thesis 1988). 